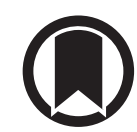

CrossMark

\title{
A rare case of sarcoidosis-associated pulmonary hypertension in a patient exposed to silica
}

\begin{abstract}
To the Editor:
Pulmonary hypertension $(\mathrm{PH})$ is a significant and well-recognised complication of sarcoidosis that markedly increases mortality. The current European Society of Cardiology (ESC)/European Respiratory Society (ERS) guidelines classify sarcoidosis-associated PH in group 5 [1]. Indeed, the pathophysiology is poorly understood and multiple mechanisms may contribute to its pathogenesis. The extrinsic compression of the large pulmonary arteries and/or veins by fibrosing mediastinitis is an uncommon mechanism of sarcoidosis-associated PH. Recognition of PH complicating sarcoidosis-associated fibrosing mediastinitis may be challenging, as the radiological features may suggest other causes of PH. Here, we report the case of a patient with sarcoidosis-associated fibrosing mediastinitis with resultant $\mathrm{PH}$ caused by exposure to silica and mimicking chronic thromboembolic pulmonary hypertension (CTEPH).
\end{abstract}

A 66-year-old man with a recent diagnosis of PH was referred to our centre for a second opinion. He had been a dental technician for 35 years and through this he had been exposed to crystalline silica dust. He had been officially declared for occupational exposure to silica and there was no history of beryllium exposure. His past medical history included HIV infection diagnosed 25 years earlier and treated with highly active antiretroviral therapy (HAART) with a CD4 count $>400 \mathrm{cells} \cdot \mathrm{mm}^{-3}$, dyslipidaemia, systemic hypertension and deep vein thrombosis without pulmonary embolism 20 years before admission. There was no history of tuberculosis. The patient reported progressive exertional dyspnoea for 4 years. Echocardiography suggested $\mathrm{PH}$ with an estimated systolic pulmonary arterial pressure of $55 \mathrm{mmHg}$ and normal left ventricular function. A ventilation/perfusion $\left(V^{\prime} / Q^{\prime}\right)$ lung scan showed multiple bilateral segmental mismatched perfusion defects suggestive of CTEPH (figure 1a). Pulmonary function tests showed an obstructive ventilatory defect with a forced expiratory volume in $1 \mathrm{~s}(\mathrm{FEV} 1) /$ forced vital capacity (FVC) ratio of $52 \%$ predicted, FEV1 $88 \%$ predicted, normal FVC and total lung capacity, and normal diffusing capacity of the lung for carbon monoxide. Arterial blood gases in room air showed an arterial oxygen tension of $79 \mathrm{mmHg}$ and an arterial carbon dioxide tension of $33 \mathrm{mmHg}$. Chest radiography showed left lower lobe atelectasis. A computed tomography (CT) scan confirmed left lower lobe atelectasis and showed bilateral hilar and mediastinal lymphadenopathy. Fluorine-18-fluorodeoxyglucose positron emission tomography (FDG-PET)/CT showed hypermetabolic activity in the mediastinal and hilar lymph nodes (maximum standardised uptake value 4.7). Fibre-optic bronchoscopy did not show endobronchial abnormalities. Bronchoalveolar lavage revealed an increased lymphocyte count (20\%) with a CD4/CD8 ratio of 2.5. Transbronchial biopsies under endobronchial ultrasound showed noncaseating granulomas consistent with sarcoidosis, and electron microscopy identified fragments of silica. Acid-fast stain was negative. The QuantiFERON-TB Gold test (QIAGEN, Chadstone, Australia) gave a negative result. Diagnosis of sarcoidosis-associated PH was suspected, but CTEPH could not be excluded. Therefore, the patient was referred to the French Referral Centre for Pulmonary Hypertension for additional evaluation. At admission, the patient was in New York Heart Association (NYHA) functional class II. He had excessive sputum production and a cough. Physical examination was unremarkable with no signs of right heart failure. Right heart catheterisation showed a mean pulmonary artery pressure of $27 \mathrm{mmHg}$, a right atrial pressure of $6 \mathrm{mmHg}$, a pulmonary capillary wedge pressure of $8 \mathrm{mmHg}$, a cardiac output of $6.3 \mathrm{~L} \cdot \mathrm{min}^{-1}$ (cardiac index $3.17 \mathrm{~L} \cdot \mathrm{min}^{-1} \cdot \mathrm{m}^{-2}$ ), and a pulmonary vascular resistance (PVR) of $3 \mathrm{Wood}$ units. High-resolution computed tomography (HRCT) of the chest showed soft tissue which had diffusely infiltrated the mediastinum and caused an encasement and narrowing of the large pulmonary arteries as well as the right and left inferior lobar bronchus (figure 1c). Pulmonary angiography confirmed the extrinsic stenoses of the large pulmonary arteries, which were most prominent on the left, and excluded the diagnosis of CTEPH (figure $1 \mathrm{~b}$ ). A diagnosis of $\mathrm{PH}$ complicating sarcoidosis-associated fibrosing mediastinitis caused by exposure to silica was retained as the most plausible diagnosis.

Due to the FDG-PET CT findings suggesting active disease, we decided to start systemic corticosteroids at a dose of $0.75 \mathrm{mg} \cdot \mathrm{kg}^{-1}$ daily. 3 months after starting steroids the patient was stable in NYHA functional class II but had reduced cough and sputum production. 
a)

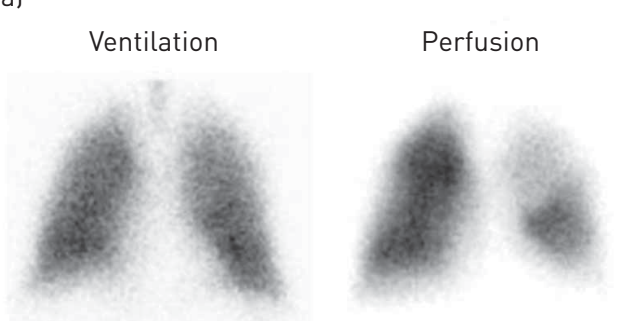

Anterior view

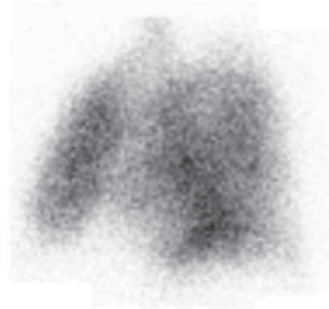

Left oblique anterior view

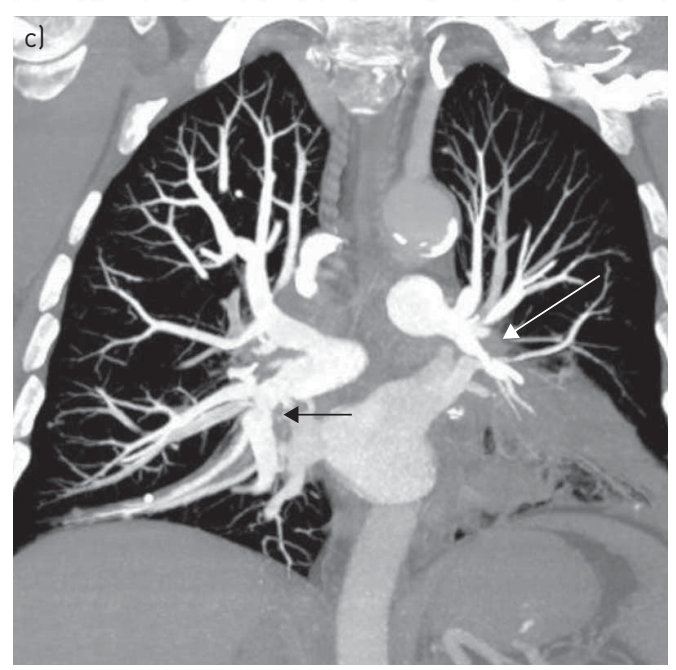

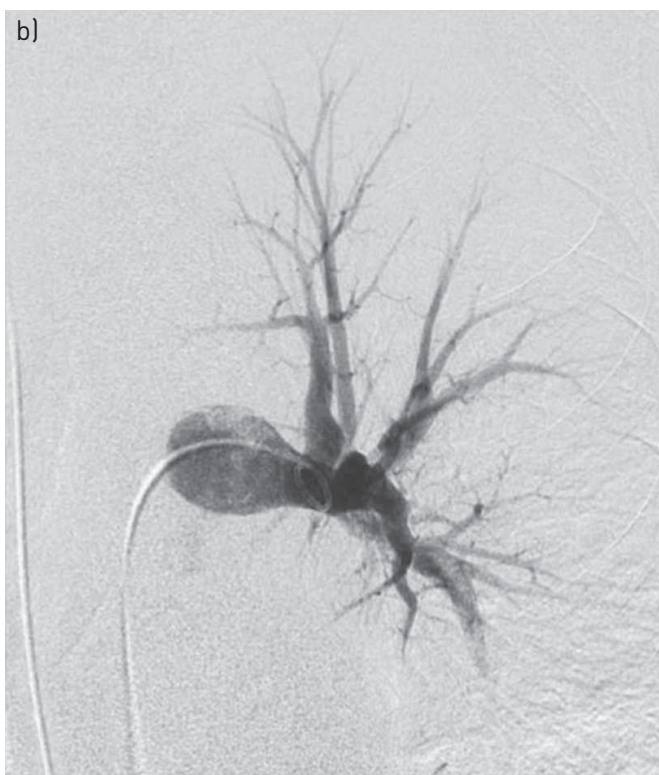

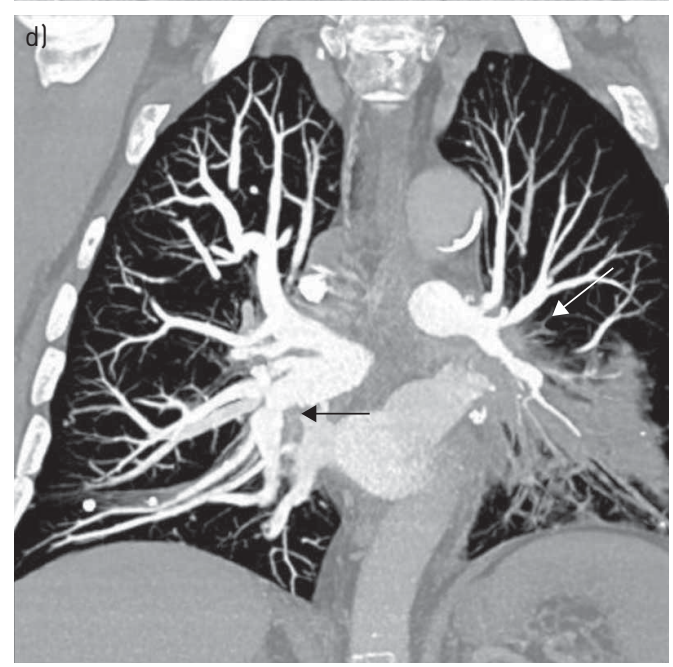

FIGURE 1 a) Ventilation/perfusion lung scan showing multiple mismatched perfusion defects in the left lower lobe and the left upper lobe. b) Conventional pulmonary angiography. The left anterior view shows an extrinsic compression of the left pulmonary artery. c, d) Contrast-enhanced high-resolution computed tomography of the chest with pulmonary arteries reformatted with maximal-intensity-projection in coronal views. c) The coronal view at baseline shows a right and left mediastinal and hilar soft tissue attenuation mass causing an encasement of the proximal right and left pulmonary arteries (arrows). d) The coronal view after 3 months of corticosteroids shows a mild decrease in the extrinsic compression of the right pulmonary artery whereas the stenosis of the left pulmonary artery remains unchanged (arrows).

Haemodynamics were improved with cardiac output increased to $7.6 \mathrm{~L} \cdot \mathrm{min}^{-1}$ (cardiac index 3.5 $\mathrm{L} \cdot \mathrm{min}^{-1} \cdot \mathrm{m}^{-2}$ ) and PVR decreased to 1.8 Wood units, whereas mean pulmonary artery pressure remained stable. Repeat HRCT of the chest showed a mild improvement in the narrowing of the right pulmonary artery, whereas the stenosis of the left pulmonary artery remained unchanged (figure 1d).

This is the first reported case of sarcoidosis-associated fibrosing mediastinitis caused by exposure to silica, complicated by $\mathrm{PH}$, and mimicking CTEPH.

Pulmonary arterial hypertension is a well known complication of HIV infection that can occur in patients with CD4 counts $>200$ cells $\cdot \mathrm{mm}^{-3}$ who are treated with HAART. However, in our case report the $V^{\prime} / Q^{\prime}$ lung scan findings as well as CT and pulmonary angiography, showing vascular and bronchial encasement and narrowing, were suggestive of another cause. Thus, the most plausible diagnosis was $\mathrm{PH}$ secondary to fibrosing mediastinitis.

A sarcoidosis like granulomatous reaction, induced by immune restoration was also ruled out. In the literature, cases of sarcoidosis in HIV patients usually appear $\sim 9$ months after HIV diagnosis, and are rare. 
In fact, sarcoidosis is an immune disease in which CD4 lymphocytes with a T-helper cell (Th)1 response play a crucial role. In our case the HIV was stable, and HAART had been started 25 years earlier. Risk factors of immune reconstitution inflammatory syndrome were absent (i.e. CD4 count $>400 \mathrm{cells} \cdot \mathrm{mm}^{-3}$ and indictable viral load, both of which had been stable for several years), and diagnostic criteria were absent (decreased viral load and increased CD4 count). So a granulomatous disorder associated with immune restoration in this HIV patient receiving HAART, was unlikely.

Sarcoidosis is a multisystem granulomatous disease, the pathogenesis of which comprises several sequential immunological events resulting in granuloma formation. These events involve exposure to one or several, so far, unidentified antigen(s), which leads to activation of macrophages and T-cell immunity against the antigen(s) and to the formation of granuloma. Infectious, organic and non-organic agents are considered plausible antigens that may trigger sarcoidosis [2].

Silica may, therefore, have contributed to the formation of granuloma as well as to the persistent granulomatous inflammation in the mediastinum and the inappropriate fibrosis seen in our patient. Indeed, an association between exposure to silica and sarcoidosis has been reported in one study [3]. However, this association was not observed in a large study using data from an occupational mortality surveillance programme performed in Iceland [4]. Distinction between silicosis and sarcoidosis due to silica exposure remains unclear in the literature, but some mechanisms are similar. Cooperation between CD4 T-lymphocytes and antigen presenting cells with inflammatory cytokine production play an important role in development of silicosis and sarcoidosis. In this case the silica exposure was confirmed on transbronchial biopsies, there were no florid radiological findings in favour of silicosis and the disease responded to treatment with corticosteroids. Classically silicosis is unresponsive to steroids and this is an argument in favour of the diagnosis of sarcoidosis associated with silica exposure. This case adds data in favour of the concept of exposure to inhaled antigens (inorganic) leading to the development of sarcoidosis.

Fibrosing mediastinitis is a rare manifestation of mediastino-pulmonary involvement that can be observed in sarcoidosis. Its frequency is unknown, but in a review by SCHOWENGERDT et al. [5] sarcoidosis accounted for $11 \%$ of confirmed cases of granulomatous and fibrous mediastinitis. It is a severe disorder characterised by the proliferation of locally invasive fibrous tissue in the mediastinum, which causes extrinsic obstruction or narrowing of large vessels (mainly pulmonary arteries and/or veins) and/or bronchi and leads to the development of $\mathrm{PH}$ and/or obstructive ventilatory defects in some patients [6]. The exact frequency of $\mathrm{PH}$ is unknown but seems to be rare [6].

Diagnosis of sarcoidosis-associated fibrosing mediastinitis may be challenging because its radiological features can suggest CTEPH by the presence of mismatched segmental perfusion defects on the $V^{\prime} / Q^{\prime}$ lung scan that can be observed in this setting [7]. HRCT scans of the chest with the use of maximal-intensity-projection are helpful to recognise the process, but pulmonary angiography may be required for the most difficult cases [8]. If the diagnosis is suspected, patients must be screened for $\mathrm{PH}$ by echocardiography. There is no established treatment for $\mathrm{PH}$ complicating sarcoidosis-associated fibrosing mediastinitis. Some improvements have been reported with the use of corticosteroids [7, 9], but other studies did not show any beneficial effect [10]. The use of $\mathrm{PH}$ specific therapies is not supported by current evidence. Surgical and/or endovascular procedures with stent placement have been proposed in highly selected patients but these options are associated with high morbidity/mortality [11, 12]. Lung or heartlung transplantation should be considered for patients with severe $\mathrm{PH}$, as mortality is high in these patients.

In conclusion, clinicians should be aware of this rare and severe cause of $\mathrm{PH}$ that can masquerade as $\mathrm{CTEPH}$ and in which HRCT of the chest and pulmonary angiography are crucial to confirm the diagnosis.

@ERSpublications

Mechanism of sarcoidosis-associated pulmonary hypertension may be challenging and requires high-quality imaging http://ow.ly/UvJPh

Delphine Bourlier ${ }^{1,2,3}$, Caroline $\mathrm{O}^{\prime}$ Connell ${ }^{1,2,3}$, David Montani ${ }^{1,2,3}$, Laurent Savale ${ }^{1,2,3}$, Andrei Seferian ${ }^{1,2,3}$, Florence Parent $^{1,2,3}$, Marc Humbert ${ }^{1,2,3}$, Gérald Simonneau ${ }^{1,2,3}$, Olivier Sitbon ${ }^{1,2,3}$ and Xavier Jaïs ${ }^{1,2,3}$

${ }^{1}$ Univ Paris-Sud, Faculté de Médecine, Université Paris-Saclay, Le Kremlin-Bicêtre, France. ${ }^{2}$ AP-HP, Service de Pneumologie, Hôpital Bicêtre, Le Kremlin-Bicêtre, France. ${ }^{3}$ INSERM UMR_S 999, Centre Chirurgical Marie Lannelongue, Le Plessis Robinson, France.

Correspondence: Delphine Bourlier, Service de pneumologie de soins intensifs, 78 avenue du Général Leclerc, Le Kremlin Bicêtre, 94270, France. E-mail: bourlier.delph@gmail.com

Received: Sept 182015 | Accepted after revision: Nov 042015

Conflict of interest: Disclosures can be found alongside the online version of this article at err.ersjournals.com

Provenance: Submitted article, peer reviewed. 


\section{References}

1 Galiè N, Humbert M, Vachiery J-L, et al. 2015 ESC/ERS Guidelines for the diagnosis and treatment of pulmonary hypertension. Eur Respir J 2015; 46: 903-975.

2 Iannuzzi MC, Rybicki BA, Teirstein AS. Sarcoidosis. N Engl J Med 2007; 357: 2153-2165.

3 Newman KL, Newman LS. Occupational causes of sarcoidosis. Curr Opin Allergy Clin Immunol 2012; 12: $145-150$.

4 Rafnsson V, Ingimarsson O, Hjalmarsson I, et al. Association between exposure to crystalline silica and risk of sarcoidosis. Occup Environ Med 1998; 55: 657-660.

5 Schowengerdt CG, Suyemoto R, Main FB. Granulomatous and fibrous mediastinitis. A review and analysis of 180 cases. J Thorac Cardiovasc Surg 1969; 57: 365-379.

6 Berry DF, Buccigrossi D, Peabody J, et al. Pulmonary vascular occlusion and fibrosing mediastinitis. Chest 1986; 89: 296-301.

7 Hasegawa K, Ohno S, Takada M, et al. Sarcoidosis complicated with major pulmonary artery obstruction and stenosis. Intern Med 2012; 51: 2775-2780.

8 Devaraj A, Griffin N, Nicholson AG, et al. Computed tomography findings in fibrosing mediastinitis. Clin Radiol 2007; 62: 781-786.

9 Toonkel RL, Borczuk AC, Pearson GD, et al. Sarcoidosis-associated fibrosing mediastinitis with resultant pulmonary hypertension: a case report and review of the literature. Respiration 2010; 79: 341-345.

10 Peikert T, Colby TV, Midthun DE, et al. Fibrosing mediastinitis: clinical presentation, therapeutic outcomes, and adaptive immune response. Medicine (Baltimore) 2011; 90: 412-423.

11 Brown ML, Cedeño AR, Edell ES, et al. Operative strategies for pulmonary artery occlusion secondary to mediastinal fibrosis. Ann Thorac Surg 2009; 88: 233-237.

12 Doyle TP, Loyd JE, Robbins IM. Percutaneous pulmonary artery and vein stenting: a novel treatment for mediastinal fibrosis. Am J Respir Crit Care Med 2001; 164: 657-660. 